\title{
A model to calculate solar radiation fluxes on the Martian surface
}

\author{
Álvaro Vicente-Retortillo ${ }^{1,3, *}$, Francisco Valero ${ }^{1}$, Luis Vázquez ${ }^{2}$, and Germán M. Martínez ${ }^{3}$ \\ 1 Departamento de Física de la Tierra, Astronomía y Astrofísica II, Facultad de Ciencias Físicas, Universidad Complutense, \\ Madrid, Spain \\ *Corresponding author: alvarodv@ucm.es \\ 2 Departamento de Matemática Aplicada, Facultad de Informática, Instituto de Matemática Interdisciplinar, \\ Universidad Complutense, Madrid, Spain \\ 3 Department of Atmospheric, Oceanic and Space Sciences, University of Michigan, Ann Arbor, USA
}

Received 25 March 2015 / Accepted 15 September 2015

\begin{abstract}
We present a new comprehensive radiative transfer model to study the solar irradiance that reaches the surface of Mars in the spectral range covered by MetSIS, a sensor aboard the Mars MetNet mission that will measure solar irradiance in several bands from the ultraviolet (UV) to the near infrared (NIR). The model includes up-to-date wavelength-dependent radiative properties of dust, water ice clouds, and gas molecules. It enables the characterization of the radiative environment in different spectral regions under different scenarios. Comparisons between the model results and MetSIS observations will allow for the characterization of the temporal variability of atmospheric optical depth and dust size distribution, enhancing the scientific return of the mission. The radiative environment at the Martian surface has important implications for the habitability of Mars as well as a strong impact on its atmospheric dynamics and climate.
\end{abstract}

Key words. Spectral irradiance - Modelling - Surface - Planets - Missions

\section{Introduction}

Advances in space exploration require a deeper knowledge of the environments of the bodies of the solar system. The characterization of the solar radiation reaching planetary environments has become crucial to address aspects related to their habitability and physical processes, resulting in one of the main topics under study in planetary space weather (Lilensten et al. 2014).

Here we focus on the solar radiation reaching the Martian surface. Solar ultraviolet radiation is very important for habitability because it is linked to biological effects and potential survival of organisms at the surface of Mars (CórdobaJabonero et al. 2003; Patel et al. 2004). Additionally, surface solar radiation measured at different spectral bands can provide information about the concentration of atmospheric dust and various Martian atmospheric components such as $\mathrm{O}_{3}$, as well as about their variations from diurnal to annual scales (Perrier et al. 2006; Vázquez et al. 2007; Zorzano et al. 2009; GómezElvira et al. 2012; Lefèvre et al. 2014). Furthermore, the spatial distribution of the absorbed radiation in the atmosphere has an important effect on atmospheric temperatures (Madeleine et al. 2011) and dynamics (Read \& Lewis 2004). Also, the solar radiation is the main term of the Martian surface energy budget (Martínez et al. 2014), which in turn controls the diurnal evolution of ground temperature (Savijärvi \& Kauhanen 2008; Martínez et al. 2009) and thus the thermodynamic processes in the Martian Planetary Boundary Layer (PBL) (Martínez et al. 2011; Petrosyan et al. 2011).

The Rover Environmental Monitoring Station (REMS) UV sensor on board the Mars Science Laboratory (MSL) mission measures the solar radiation at the surface of Gale crater $\left(4.6^{\circ} \mathrm{S}\right)$ in six bands between 200 and $380 \mathrm{~nm}$. In support of the REMS UV sensor, and to extend its measuring range, the Mars MetNet Mission (http://metnet.fmi.fi) includes a Solar Irradiance Sensor (MetSIS) as part of its payload, which has been designed to measure solar radiation at the Martian surface in several bands up to $1100 \mathrm{~nm}$. Most of the MetSIS channels have a hemispherical field of view and are designed to be pointing to the zenith, measuring therefore the solar irradiance at the surface.

Here we present a new comprehensive radiative transfer model that calculates the spectral solar irradiance from wavelength-dependent radiative parameters, enabling calculations of solar radiation fluxes in different spectral regions covered by MetSIS and REMS. The various results of the model provide information about the radiative environment at the Martian surface under typical and extreme scenarios, and allow comparisons between model results and in situ measurements, improving the scientific return of present and future missions to Mars.

In Section 2, we describe the radiative transfer model, hereinafter COMIMART (COmplutense and MIchigan MArs Radiative Transfer model). In Section 3, we validate COMIMART using as a reference solar fluxes calculated by the DISORT radiative transfer algorithm (Stamnes et al. 1988). In Section 4, we show important results derived from the model to characterize the radiative environment at the Martian surface: first, we present an analysis of the contributions of the individual atmospheric components to the total optical depth and perform sensitivity studies for scenarios that cover most of the possible atmospheric compositions at low and mid-latitudes; then, we analyze the diurnal evolution of integrated solar fluxes in the 
MetSIS band 200-1100 nm under different scenarios and compare them with fluxes calculated in the full shortwave spectral range; finally, we study spectral irradiances in the range 200-1100 nm also under different scenarios, and describe a method to estimate the optical depth and particle size distribution from the model and in situ measurements in different bands. In Section 5, we summarize our results and discuss their importance.

\section{Description of the model}

The solar radiation at the top of the atmosphere (TOA) and the radiative properties of the atmosphere govern the solar flux that reaches the surface of Mars. The calculation of the solar radiation at the TOA and the determination of the radiative properties of the Martian atmosphere based on updated wavelength-dependent radiative properties of its components are described below.

\subsection{Solar spectrum at the top of the atmosphere}

The spectral irradiance that reaches a horizontal surface at the TOA as a function of the latitude and time of the year can be determined using the expression

$$
\begin{aligned}
E=E_{m} & {\left[\sin \varepsilon \sin L_{s} \sin \phi+\left(1-\sin ^{2} \varepsilon \sin ^{2} L_{s}\right)^{1 / 2}\right.} \\
& \left.\times \cos \phi \cos \frac{2 \pi t}{P}\right]\left[\frac{1+e \cos \left(L_{s}-L_{s, p}\right)}{1-e^{2}}\right]^{2},
\end{aligned}
$$

where $E_{m}$ represents the spectral irradiance at the mean distance between the Sun and Mars (1.52 AU), $e=0.0934$ is the eccentricity of the orbit of Mars, $L_{s, p}=251^{\circ}$ is the solar longitude at the perihelion, $\varepsilon=25.2^{\circ}$ is the Martian obliquity, $P=88,775 \mathrm{~s}$ is the length of a sol (a Martian day), $\phi$ is the latitude, and $t$ is the time measured in seconds from local noon (Haberle et al. 1993; Patel et al. 2002). In COMIMART, $E_{m}$ is determined from the 2000 American Society for Testing and Materials (ASTM) Standard Extraterrestrial Solar Spectrum Reference E-490-00 (http://rredc. nrel.gov/solar/spectra/am0).

The first bracket on the right-hand side of Eq. (1) represents the cosine of the solar zenith angle, while the second one shows the ratio of the mean distance between the Sun and Mars to the distance at a given orbital position. By using Eq. (1) we are assuming that the Sun is a point source, a standard approximation for radiative transfer studies on the Earth due to the small errors involved except during sunrise or sunset (Stamnes et al. 2000), which become even smaller for Mars due to the larger distance to the Sun.

\subsection{Radiative properties of the Martian atmosphere}

The different components of the Martian atmosphere interact with the solar radiation by means of absorption and scattering processes. The components considered in this model are dust, water ice clouds, and gas molecules $\left(\mathrm{CO}_{2}, \mathrm{~N}_{2}, \mathrm{Ar}, \mathrm{O}_{2}, \mathrm{O}_{3}\right.$, and $\mathrm{H}_{2} \mathrm{O}$ ). These components are denoted with the subscripts $d, c$, and $g$.

In order to obtain the amount of radiation that reaches the surface, the total atmospheric optical depth $\tau$ is calculated from the individual contributions of each atmospheric component as

$$
\tau(\lambda)=\tau_{g}(\lambda)+\tau_{d}(\lambda)+\tau_{c}(\lambda) .
$$

The optical depth of each component can be separated into two terms: one accounting for scattering (indicated with the subscript $s$ ) and the other for absorption (denoted with the subscript $a$ ). Following this notation, Eq. (2) can be rewritten as

$$
\begin{aligned}
\tau(\lambda)= & \tau_{g s}(\lambda)+\tau_{d s}(\lambda)+\tau_{c s}(\lambda)+\tau_{g a}(\lambda)+\tau_{d a}(\lambda) \\
& +\tau_{c a}(\lambda) .
\end{aligned}
$$

A description of the calculation of the terms of Eq. (3) is provided below, beginning with dust, continuing with clouds, and ending with the terms related to the gas molecules.

Among the various Martian atmospheric constituents, dust has the greatest impact on the absorption and scattering of solar radiation. In order to quantify the result of its interaction with solar radiation, three radiative parameters are needed: the extinction efficiency, $Q_{\text {ext }, d}(\lambda)$, defined as the extinction crosssection (the sum of the scattering and absorption crosssections) divided by the projected surface area of the spherical particle; the single scattering albedo, $\omega_{0, d}(\lambda)$, defined as the ratio between scattering and extinction coefficients; and the asymmetry factor, $g_{d}(\lambda)$, defined as the first moment of the phase function, which represents the probability of scattering in any given direction.

In COMIMART, we calculate updated wavelengthdependent values of these three parameters by using the Mie theory and the refractive indices presented by Wolff et al. (2009) and Wolff et al. (2010). These three parameters also depend on the size distribution of the particles. Here we assume a log-normal size distribution, characterized by the effective radius, $r_{\text {eff }}$, and the effective variance, $v_{\text {eff }}$ (Hansen \& Travis 1974), and select standard values of $r_{\mathrm{eff}}=1.5 \mu \mathrm{m}$ (Clancy et al. 2003; Wolff \& Clancy, 2003; Kahre et al. 2006; Madeleine et al. 2011) and $v_{\text {eff }}=0.3$ (Rannou et al. 2006; Madeleine et al. 2011). The reader is referred to Section 4.3 for a discussion of the impact of choosing different size distributions on the calculation of the spectral irradiance.

Madeleine et al. (2011) show that the opacity of the atmosphere is directly proportional to the extinction efficiency. Thus, by using the value of the optical depth at any particular wavelength as a reference, we calculate the dust opacity $\tau_{d}(\lambda)$ at any other wavelength from the corresponding value of $Q_{\text {ext }, d}(\lambda)$. We have chosen $880 \mathrm{~nm}$ as a reference in COMIMART to enable a direct comparison to the measurements taken by the Pancam cameras at the locations of the Mars Exploration Rovers (Lemmon et al. 2015) and by the Mastcam cameras at the MSL site; we denote the dust optical depth at this wavelength by $\tau_{d, 880}$. The model enables the calculation of the two contributions (scattering and absorption) to $\tau_{d}$. The scattering optical depth due to dust particles in Eq. (3) is obtained by multiplying $\tau_{d}(\lambda)$ by $\omega_{0, d}(\lambda)$.

Solar radiation also interacts with water ice clouds. In order to quantify their effect, we calculate the water ice radiative properties $\left(Q_{\text {ext }, c}(\lambda), \omega_{0, c}(\lambda)\right.$, and $\left.g_{c}(\lambda)\right)$ from the refractive indices given by Warren (1984). In this case, we select a log-normal size distribution with $r_{\text {eff }}=3 \mu \mathrm{m}$ (Wolff \& Clancy 2003) and $v_{\text {eff }}=0.1$ (Wolff \& Clancy 2003; Madeleine et al. 2012). In order to obtain $\tau_{c}(\lambda)$, we follow the same procedure as for the dust, but selecting $825 \mathrm{~cm}^{-1}$ (roughly $12.1 \mu \mathrm{m}$ ) as 
the reference wavelength to simplify the comparison to Thermal Emission Spectrometer (TES) results (Smith 2004). Thus, we obtain $\tau_{c}(\lambda)$ as

$$
\tau_{c}(\lambda)=\frac{Q_{\mathrm{ext}, c}(\lambda)}{Q_{\mathrm{ext}, c, 12.1}\left(1-\omega_{0, c 12.1}\right)} \tau_{c a, 12.1},
$$

where $\tau_{c a, 12.1}$ stands for the TES absorption optical depth. We have followed the same scheme as in the dust case to calculate the absorption and scattering cloud optical depths at each wavelength.

The interaction of gas molecules with solar radiation is quantified by means of their scattering and absorption crosssections. The total optical depth due to scattering by gas molecules is given by the sum of the scattering optical depths of $\mathrm{CO}_{2}, \mathrm{~N}_{2}, \mathrm{Ar}$, and $\mathrm{O}_{2}$, which are calculated multiplying their column abundance $N$ by their Rayleigh scattering cross-section $\sigma_{s}$ as follows

$$
\tau_{g s}=\sigma_{s, \mathrm{CO}_{2}} N_{\mathrm{CO}_{2}}+\sigma_{s, \mathrm{~N}_{2}} N_{\mathrm{N}_{2}}+\sigma_{s, \mathrm{Ar}_{\mathrm{r}}} N_{\mathrm{Ar}}+\sigma_{s, \mathrm{O}_{2}} N_{\mathrm{O}_{2}} .
$$

Note that scattering by $\mathrm{O}_{3}$ and $\mathrm{H}_{2} \mathrm{O}$ is not considered because these molecules are variable trace species in the Martian atmosphere, and thus we can consider their effect on Rayleigh scattering negligible. The column abundance is obtained using the expression

$$
N=\frac{p \times \mathrm{Av}}{M \times g^{*}} n_{\mathrm{rel}},
$$

where $p$ represents the pressure at the surface, Av is the Avogadro's number, $M$ is the molecular mass of the gas, $g^{*}$ is the gravity, and $n_{\text {rel }}$ is the relative abundance of the gas in the atmosphere. The scattering cross-section is obtained as

$$
\sigma_{s}(\lambda)=\frac{24 \pi^{3}}{\lambda^{2} N_{s}^{2}}\left(\frac{n^{2}(\lambda)-1}{n^{2}(\lambda)+2}\right)^{2} F_{k}(\lambda),
$$

where $N_{s}$ represents the number of molecules per unit of volume, $n$ is the refractive index, and $F_{k}$ is the King correction factor (Sneep \& Ubachs 2005). Refractive indices for $\mathrm{CO}_{2}$ and $\mathrm{O}_{2}$ are taken from Sneep \& Ubachs (2005), while for $\mathrm{N}_{2}$ and Ar they are taken from Weber (2003) and references herein. King correction factors for $\mathrm{CO}_{2}, \mathrm{O}_{2}, \mathrm{~N}_{2}$, and Ar are taken from Sneep \& Ubachs (2005).

Absorption by gas molecules is calculated from their absorption cross-sections when an absorption continuum is observed for a particular component. In addition to the absorption continua, some gas molecules of the Martian atmosphere, especially $\mathrm{CO}_{2}$ and $\mathrm{H}_{2} \mathrm{O}$, show absorption lines that can be characterized individually in the shortwave range (Rothman et al. 2013). We have not included these lines in the model because they have a negligible effect in the 200-1100 nm range, and still a very small effect in the remaining range of the shortwave range (the reader is referred to Sect. 3.1.1 for further details on this assumption). Taking this into account, the total optical depth due to absorption by gas molecules is calculated from

$$
\tau_{g a}=\sigma_{a, \mathrm{CO}_{2}} N_{\mathrm{CO}_{2}}+\sigma_{a, \mathrm{O}_{2}} N_{\mathrm{O}_{2}}+\sigma_{a, \mathrm{O}_{3}} N_{\mathrm{O}_{3}}+\sigma_{a, \mathrm{H}_{2} \mathrm{O}} N_{\mathrm{H}_{2} \mathrm{O}},
$$

where $\sigma_{a}$ represents the absorption cross-section of each gas. Since the mixing ratios of $\mathrm{O}_{3}$ and $\mathrm{H}_{2} \mathrm{O}$ are not vertically uniform, their column abundances are not obtained from Eq. (6), but introduced as model inputs.

In the present Martian atmosphere, $\mathrm{CO}_{2}$ is the gas with the greatest impact on solar radiation. $\mathrm{CO}_{2}$ absorption cross-sections have been included for the spectral range between 122 and $202 \mathrm{~nm}$ (Lewis \& Carver 1983; Ityaksov et al. 2008), this last value representing the wavelength at which $\mathrm{CO}_{2}$ absorption approximately begins to be negligible (Ityaksov et al. 2008). The high values of the absorption cross-section, together with the $\mathrm{CO}_{2}$ abundance in the Martian atmosphere, produce a cut at roughly $200 \mathrm{~nm}$ in the solar spectrum that reaches the Martian surface. The temperature dependence of the cross-sections measured in the range from $200 \mathrm{~K}$ to $370 \mathrm{~K}$ by Lewis \& Carver (1983) has been included in COMIMART, but due to the low spectral irradiance at these wavelengths and the aforementioned cut in the spectrum, its impact on the solar flux at the surface is small.

Besides $\mathrm{CO}_{2}$, there are other radiatively active gas molecules, although they are present in small amounts in the Martian atmosphere. We have included $\mathrm{O}_{3}$ cross-sections in the ranges 214-1100 $\mathrm{nm}$ and 203-293 K (Serdyuchenko et al. 2014) and $\mathrm{O}_{2}$ cross-sections in the ranges 176$242.5 \mathrm{~nm}$ and 200-300 K (Frederick \& Mentall 1982). Despite the cut in the spectrum produced by $\mathrm{CO}_{2}$, we have included $\mathrm{H}_{2} \mathrm{O}$ cross-sections in the range 182-197 nm (Parkinson \& Yoshino 2003) to allow studies in scenarios with very different relative molecular abundances. $\mathrm{H}_{2} \mathrm{O}_{2}$ molecule presents absorption in the UV region with relatively high absorption cross-sections (Lin et al. 1978), but its effect has not been considered here because its mixing ratio shows typical values as low as $10^{-8}$ (Encrenaz et al. 2012).

Once the total optical depth attributable to all the atmospheric components is obtained, effective values for the single scattering albedo and for the asymmetry factor are determined. The former is given by the ratio between the total scattering optical depth (the sum of the first three terms of the right-hand side of Eq. (3)) and the total optical depth (Eq. (2)); the latter is obtained from the equation

$$
g=\frac{\tau_{d s} g_{d}+\tau_{c s} g_{c}}{\tau_{g s}+\tau_{d s}+\tau_{c s}},
$$

where the assumption of isotropic molecular scattering has been taken.

\subsection{Calculating solar fluxes}

We calculate solar fluxes in any given band by using the deltaEddington approximation, which is based on the two-stream Eddington approximation for multiple scattering plane-parallel atmospheres, except that it includes a delta adjustment in the phase function to improve the accuracy when the asymmetry factor is high (Joseph et al. 1976). This is the case of the Martian aerosols, particularly in the UV spectral region. In the delta-Eddington approximation, the asymmetry factor, the single scattering albedo and the optical depth are transformed according to Joseph et al. (1976) as

$$
\begin{gathered}
g^{\prime}=\frac{g}{1+g}, \\
\omega^{\prime}=\omega_{0} \frac{1-g^{2}}{1-\omega_{0} g^{2}}, \\
\tau^{\prime}=\tau\left(1-\omega_{0} g^{2}\right) .
\end{gathered}
$$

After the calculation of the aforementioned radiative parameters, and assuming a single layer atmosphere (see Sect. 3.1.2 
for further details on this assumption), solar fluxes at the surface are estimated using the following set of equations (Haberle et al. 1993; Patel et al. 2002)

$$
\begin{gathered}
T=E\left[C_{1} \exp (-k \tau)\left(1+P^{\prime}\right)+C_{2} \exp (k \tau)\left(1-P^{\prime}\right)\right. \\
\left.-(\alpha+\beta-1) \exp \left(-\tau / \mu_{0}\right)\right] \\
k=\left[3\left(1-\omega_{0}\right)\left(1-g \omega_{0}\right)\right]^{1 / 2} \\
P^{\prime}=\frac{2}{3}\left[\frac{3\left(1-\omega_{0}\right)}{1-g \omega_{0}}\right]^{1 / 2} \\
\alpha=\frac{3}{4} \mu_{0} \omega_{0} \frac{1+g\left(1-\omega_{0}\right)}{1-\mu_{0}^{2} k^{2}} \\
C_{5}=1-A-P^{\prime}(1+A) \\
C_{2}=\frac{1}{2} \mu_{0} \omega_{0} \frac{1 / \mu_{0}+3 \mu_{0} g\left(1-\omega_{0}\right)}{1-\mu_{0}^{2} k^{2}} \\
C_{4}=1-A+P^{\prime}(1+A) \\
\left(1+P^{\prime}\right) C_{4} \exp (k \tau)-\left(1-P^{\prime}\right) C_{5} \exp (-k \tau) \\
\left(1+P^{\prime}\right) C_{3} \exp \left(-\tau / \mu_{0}\right)-(\alpha+\beta) C_{4} \exp (k \tau)
\end{gathered}
$$

In these expressions, $T$ is the total (direct + diffuse) flux at the surface in the selected spectral region, $\mu_{0}$ is the cosine of the solar zenith angle at the TOA, and $A$ is the surface albedo. Note that the dependence of $\tau, \omega_{0}, g$, and $A$ on wavelength is not explicitly shown.

The direct (beam) flux, $B$, is calculated using the BeerLambert law:

$$
B=E \times \exp \left(-\tau / \mu_{0}\right),
$$

where $\tau$ is the value of the atmospheric optical depth before using Eq. (12). Finally, the diffuse flux, $D$, is obtained by subtracting the direct component from the total flux at the surface.

\section{Model testing and validation}

In this section we discuss various assumptions considered in the model and then validate their results by comparing the calculated surface fluxes to those obtained using the DISORT algorithm (Stamnes et al. 1988).

\subsection{Discussion on model assumptions}

\subsubsection{Effect of individual absorption lines}

We discuss the impact of the individual $\mathrm{CO}_{2}$ and $\mathrm{H}_{2} \mathrm{O}$ lines on surface fluxes and show that it can be neglected in the spectral region between 200 and $1100 \mathrm{~nm}$, and that it is still very small in the remaining range of the shortwave range.

We quantify the impact of these individual lines by using the atmospheric radiative transfer model MODTRAN (MODerate resolution atmospheric TRANsmission; Berk et al. 1998) as follows. We introduce an aerosol-free atmospheric vertical profile based on the reference profile shown by Savijärvi et al. (2005). We obtain that when $\mu_{0}=1, \mathrm{CO}_{2}$ and $\mathrm{H}_{2} \mathrm{O}$ absorb together only about $1 \%$ of the incoming solar radiation at the top of the atmosphere. Moreover, the absorption due to these individual lines is much smaller between 200 and $1100 \mathrm{~nm}$, representing only a $0.0091 \%$ of $E$ when the Sun is at the zenith. When $\mu_{0}=0.5$, the fraction of $E$ absorbed by these lines increases because of the longer optical path, but its value remains very small $(0.016 \%)$. These results confirm that the effect of these lines on the solar flux measured at wide spectral bands in the MetSIS range is negligible. In addition, considering the effect of each individual line at the corresponding wavelength would require a very high spectral resolution, significantly slowing down the computing process.

\subsubsection{Single layer approximation}

We test the validity of the single layer approximation by using DISORT to calculate and compare fluxes at the surface under five different scenarios and for two different scattering regimes. For each scenario and regime, we first consider two layers (with dust at the bottom and ice clouds on top), and then only a single layer. As shown below, the differences are negligible from 200 to $1100 \mathrm{~nm}$ (MetSIS range).

The five scenarios are defined as follows. The first represents typical clear conditions, with $\tau_{d}=0.3$ and $\tau_{c}=0.02$, and with the Sun at the zenith $\left(\mu_{0}=1\right)$. The second scenario considers the same $\tau_{d}$ and $\mu_{0}$, but a higher cloud optical depth $\left(\tau_{c}=0.15\right)$. Scenarios 3 and 4 are like 1 and 2, respectively, but with $\mu_{0}=0.5$. Finally, scenario 5 considers the same $\tau_{c}$ and $\mu_{0}$ as 2 , but under typical dusty conditions $\left(\tau_{d}=1\right)$.

For each scenario, we select two different scattering regimes. This is because the dust single scattering albedo, the dust and water ice clouds asymmetry factor, and the surface albedo show significantly different properties in the UV (200 $400 \mathrm{~nm}$ ) and NIR (700-1100 nm) regions. In particular, the dust single scattering albedo and asymmetry factor show small spectral variability between 700 and $1100 \mathrm{~nm}$, with calculated values around 0.97 and 0.70 , respectively. Toward the UV region, the dust single scattering albedo decreases and the asymmetry factor increases. Moreover, the surface albedo also depends on the wavelength, with lower values in the UV region (Perrier et al. 2006) than in the NIR (Mustard \& Bell 1994).

In the two-layer case and for the bottom dust layer, we set representative values of $\omega_{0, d}=0.67$ and $g_{d}=0.87$ in the UV, and $\omega_{0, d}=0.97$ and $g_{d}=0.70$ in the NIR. For the top cloud layer, we set representative values of $\omega_{0, c}=1$ and $g_{c}=0.85$ in the UV, and $\omega_{0, c}=1$ and $g_{c}=0.81$ in the NIR. In the one 
Á. Vicente-Retortillo et al.: Modeling solar radiation fluxes on Mars

Table 1. Comparison between $T / E$ for a single effective atmospheric layer (denoted by the subscript 1 ) and $T / E$ for a vertically inhomogeneous atmosphere, for the conditions described in the text.

\begin{tabular}{|c|c|c|c|c|c|c|}
\hline \multirow[b]{2}{*}{ Scenario } & \multicolumn{3}{|c|}{ UV conditions } & \multicolumn{3}{|c|}{ NIR conditions } \\
\hline & $(T / E)_{1}$ & $(T / E)_{2}$ & Rel. error $(\%)$ & $(T / E)_{1}$ & $(T / E)_{2}$ & Rel. error $(\%)$ \\
\hline 1 & 0.89787 & 0.89757 & 0.033 & 0.98431 & 0.98420 & 0.011 \\
\hline 2 & 0.89211 & 0.89084 & 0.143 & 0.98153 & 0.98114 & 0.040 \\
\hline 3 & 0.78715 & 0.78660 & 0.070 & 0.90046 & 0.90046 & $<10^{-4}$ \\
\hline 4 & 0.76555 & 0.76388 & 0.219 & 0.87848 & 0.87864 & -0.018 \\
\hline 5 & 0.68327 & 0.68106 & 0.324 & 0.91125 & 0.91059 & 0.072 \\
\hline
\end{tabular}

Table 2. $T / E$ calculated using DISORT (top) and COMIMART (middle), and relative departures (bottom), for $\omega_{0}=0.67, g=0.87$, and $A=0.03$.

\begin{tabular}{|c|c|c|c|c|c|c|}
\hline \multicolumn{7}{|c|}{$\omega_{0}=0.67, g=0.87, A=0.03$} \\
\hline & & & & $\mu_{0}$ & & \\
\hline \multirow{2}{*}{\multicolumn{7}{|c|}{$\begin{array}{l}\mathbf{0 . 5} \\
T / E \text { (DISORT) }\end{array}$}} \\
\hline & & & & & & \\
\hline \multirow{6}{*}{$\tau$} & 0.3 & 0.6540 & 0.7904 & 0.8525 & 0.8798 & 0.8986 \\
\hline & 0.6 & 0.4366 & 0.6201 & 0.7221 & 0.7701 & 0.8044 \\
\hline & 1 & 0.2711 & 0.4491 & 0.5755 & 0.6415 & 0.6909 \\
\hline & 1.5 & 0.1644 & 0.3035 & 0.4314 & 0.5074 & 0.5680 \\
\hline & 2.5 & 0.0744 & 0.1465 & 0.2419 & 0.3132 & 0.3781 \\
\hline & 5 & 0.0162 & 0.0311 & 0.0595 & 0.0908 & 0.1284 \\
\hline \multicolumn{7}{|c|}{$T / E$ (COMIMART) } \\
\hline \multirow{6}{*}{$\tau$} & 0.3 & 0.6808 & 0.7955 & 0.8519 & 0.8783 & 0.8974 \\
\hline & 0.6 & 0.4735 & 0.6343 & 0.7245 & 0.7694 & 0.8028 \\
\hline & 1 & 0.3021 & 0.4706 & 0.5825 & 0.6425 & 0.6890 \\
\hline & 1.5 & 0.1819 & 0.3258 & 0.4420 & 0.5103 & 0.5658 \\
\hline & 2.5 & 0.0766 & 0.1587 & 0.2521 & 0.3172 & 0.3755 \\
\hline & 5 & 0.0133 & 0.0283 & 0.0594 & 0.0910 & 0.1263 \\
\hline \multicolumn{7}{|c|}{ Relative departure (\%) } \\
\hline \multirow{6}{*}{$\tau$} & 0.3 & 4.10 & 0.65 & -0.07 & -0.17 & -0.14 \\
\hline & 0.6 & 8.46 & 2.29 & 0.34 & -0.09 & -0.20 \\
\hline & 1 & 11.43 & 4.79 & 1.22 & 0.16 & -0.28 \\
\hline & 1.5 & 10.63 & 7.35 & 2.46 & 0.58 & -0.38 \\
\hline & 2.5 & 2.96 & 8.35 & 4.23 & 1.27 & -0.67 \\
\hline & 5 & -17.71 & -9.03 & -0.20 & 0.27 & -1.62 \\
\hline
\end{tabular}

layer case, we calculate the effective radiative parameters as described in Section 2 by considering the optical depths of each scenario and the single scattering albedo and asymmetry factor values defined above. Finally, we set values of $A=0.03$ in the UV and $A=0.25$ in the NIR.

Table 1 shows the values of $T / E$ for the single layer and two-layer cases under the five scenarios described above and for the two scattering regimes. It also shows the relative error in considering a single layer. For any possible combination of the selected scenarios and scattering regimes, the relative error is below $0.4 \%$. In particular, due to the different values of the radiative parameters of the dust and water ice clouds layers in the two scattering regimes, the departure between the single and two-layer approximation in the UV is higher than in the NIR (where it is about $10^{-2} \%$ ), but it is still very small (approximately $10^{-1} \%$ ). Therefore, the single layer approximation is valid to calculate the solar fluxes that reach the Martian surface in the MetSIS range.

\subsection{Validation of the model}

We validate COMIMART by comparing the calculated surface fluxes to those obtained using the DISORT algorithm. Values of solar fluxes at the surface depend on the optical depth, single scattering albedo, asymmetry factor, surface albedo, and zenith angle. As in Section 3.1.2, we analyze both fluxes in the UV and NIR scattering regimes for several optical depths and zenith angles, and select two sets of radiative parameters for each region, with $\omega=0.67, g=0.87$, and $A=0.03$ in the UV regime, and $\omega=0.97, g=0.70$, and $A=0.25$ in the NIR regime. The selected combination of scenarios and scattering regimes encompasses a comprehensive representation of the Mars' atmospheric radiative properties and solar geometries, ensuring a thorough validation of COMIMART.

In the DISORT model, we select a Henyey-Greenstein phase function (Henyey \& Greenstein 1941) to be consistent with the transformation of the radiative parameters in the delta-Eddington approximation shown by Eqs. (10)-(12). Also, we set the number of streams to 32 to obtain highly accurate flux values.

We show in Table 2 (UV) and Table 3 (NIR) the ratio of the flux at the surface to the flux at the top of the atmosphere $(T / E)$ calculated with DISORT (top) and with COMIMART (middle) for representative values of $\mu_{0}$ and $\tau$. We also show the relative departure, in percentage, between COMIMART and DISORT (bottom). For high values of $\mu_{0}$ and for typical optical depths 
Table 3. As in Table 2, but for $\omega_{0}=0.97, g=0.70$, and $A=0.25$.

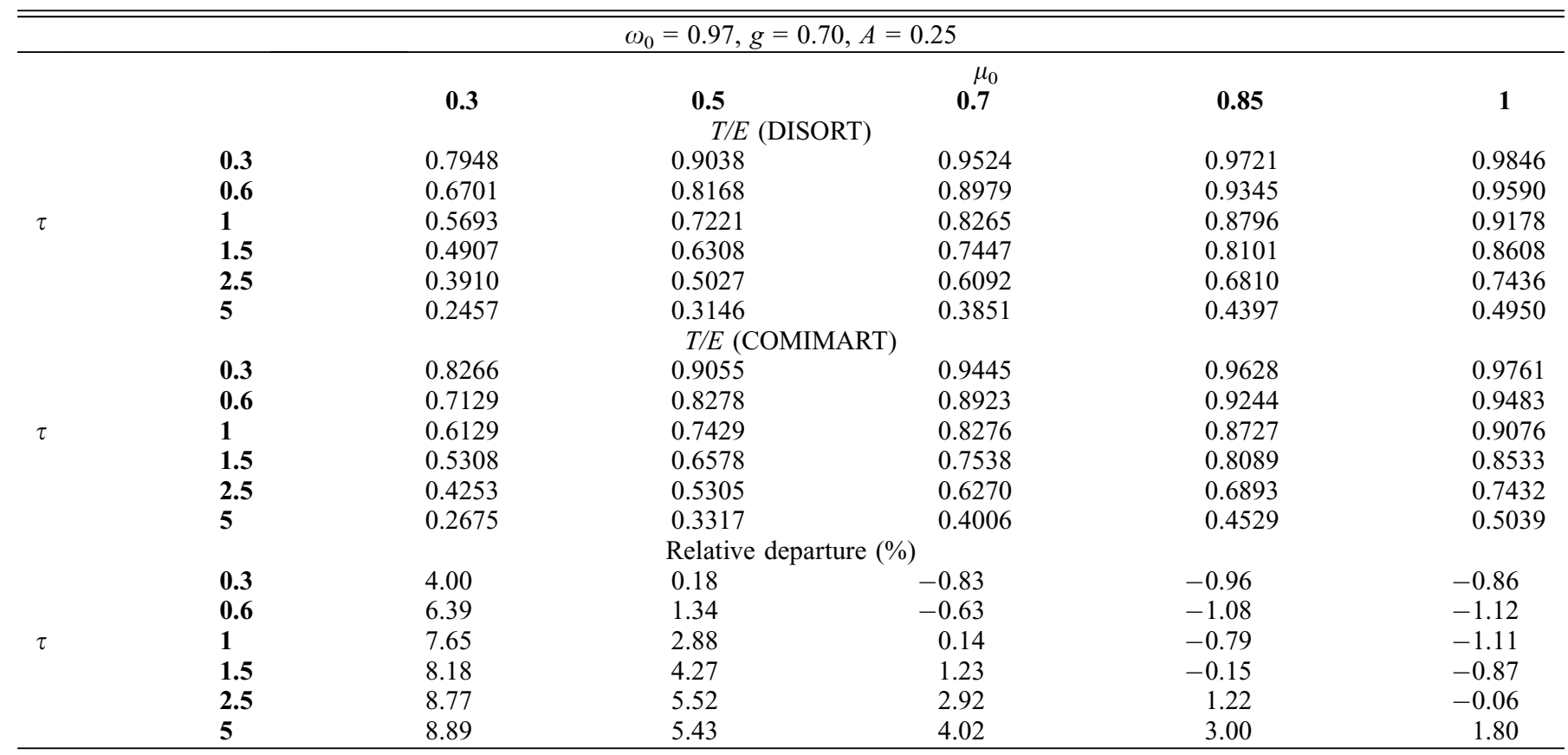

between 0.3 and 1.5 (Lemmon et al. 2015), the results obtained with both models are in excellent agreement, with absolute values of the relative departure $\sim 1 \%$ in the NIR region and even lower in the UV. For low values of $\mu_{0}$, relative departures between both models are slightly higher, with values typically between $4 \%$ and $10 \%$ regardless of the value of $\tau$. Still, the absolute departure stays comparable to that at higher solar elevations because for lower $\mu_{0}$ values, the calculated fluxes are smaller. Therefore, except at high solar zenith angles, corresponding to the times of the day close to sunrise and sunset (when the plane-parallel atmosphere assumption loses validity), the results obtained with COMIMART are accurate in the two scattering regimes.

\section{Results}

\subsection{Effect of individual atmospheric components and sensitivity studies}

Here we study the relative contribution of each atmospheric component to the total atmospheric optical depth under a typical scenario, and then we perform sensitivity studies to check the robustness of the results to changes in atmospheric conditions. These sensitivity studies show a method to estimate the optical depth from MetSIS measurements and model results.

The total optical depth is given by the sum of the individual terms in Eq. (3). We analyze the relative contribution of each of these terms under typical clear Martian atmospheric conditions at low and mid-latitudes, corresponding to the initially selected landing sites for the MetNet mission. We characterize these conditions by choosing values of dust optical depth $\tau_{d, 880}=0.3$ (Lemmon et al. 2015), water ice clouds absorption optical depth $\tau_{c a, 12.1}=0.02$ (typical value outside the aphelion cloud belt; Smith 2004; Madeleine et al. 2012), and ozone column abundance of $N_{\mathrm{O}_{3}}=1 \mu \mathrm{m}$-atm (Perrier et al. 2006).

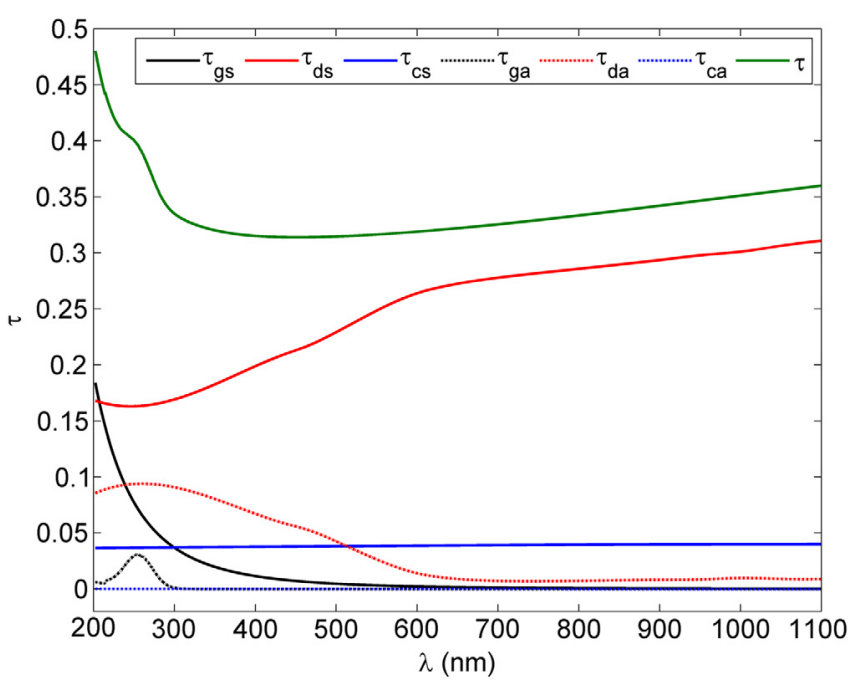

Fig. 1. Spectral behavior of the scattering and absorption optical depths (denoted by subscripts $s$ and $a$ ) of dust, water ice clouds, and gas molecules (denoted by subscripts $d, c$, and $g$ ) for $\tau_{d, 880}=0.3$, $\tau_{c a, 12.1}=0.02$, and $N_{\mathrm{O}_{3}}=1 \mu \mathrm{m}$-atm.

Figure 1 shows the spectral behavior of the six terms involved in Eq. (3). Even for a clear $\left(\tau_{d, 880}=0.3\right)$ atmosphere, dust has the greatest impact among the atmospheric constituents on the absorption and scattering of solar radiation. The values of $\tau_{d s}$ are larger than those of $\tau_{d a}$, particularly in the NIR, where values of $\tau_{d a}$ represent only about $3 \%$ of the total dust optical depth.

The effect of gas molecules on the total optical depth is significant at short wavelengths in the UV region. There, the scattering is mostly caused by $\mathrm{CO}_{2}(97.62 \%)$, followed by contributions from $\mathrm{N}_{2}(1.72 \%), \mathrm{Ar}(0.62 \%)$, and $\mathrm{O}_{2}(0.04 \%)$. The absorption optical depth of the gas molecules is noticeable 
in the range $200-300 \mathrm{~nm}$ and it shows a maximum at $255 \mathrm{~nm}$, caused by the $\mathrm{O}_{3}$ Hartley band. At wavelengths shorter than $202 \mathrm{~nm}, \tau_{g a}$ markedly increases (not shown in Fig. 1 for clarity purposes), causing the cut in the spectrum due to $\mathrm{CO}_{2}$ absorption (the absorption optical depth of $\mathrm{CO}_{2}$ is around 10 at $186 \mathrm{~nm}$ and four orders of magnitude higher at $150 \mathrm{~nm}$ ).

There is virtually no radiation absorbed by the water ice clouds in the 200-1100 $\mathrm{nm}$ region, with a maximum ratio $\tau_{c a} / \tau_{c s}$ lower than $10^{-4}\left(\omega_{0, c} \sim 1\right.$ in this spectral range). In general, the effect of water ice clouds on the total optical depth is stronger than that of gas molecules, except in the UV region, where the scattering by gas molecules exceeds that by ice clouds.

We note that the effect of variations in the ozone column abundance and of the temperature dependence of the crosssections on the calculated solar fluxes is insignificant. For instance, the surface solar flux between 200 and $310 \mathrm{~nm}$ at the equator, at noon, and at $L_{s}=270^{\circ}$ is approximately $8.18 \mathrm{~W} / \mathrm{m}^{2}$ when $N_{\mathrm{O}_{3}}=1 \mu \mathrm{m}$-atm. If we triple this value, the flux at the surface would be $8.04 \mathrm{~W} / \mathrm{m}^{2}$, and thus the decrease is lower than $1.8 \%$. The effect of temperature is even weaker: the difference between $203 \mathrm{~K}$ and $218 \mathrm{~K}$ (which is the temperature considered by Patel et al. 2002) is only of $0.0014 \%$, even for a high value of $N_{\mathrm{O}_{3}}=3 \mu \mathrm{m}$-atm. These effects are even weaker if we consider the band between 200 and $1100 \mathrm{~nm}$, where the described changes in concentration and temperature represent, respectively, $0.033 \%$ and less than a $10^{-4} \%$ of the total flux.

After having weighted the contribution of the individual atmospheric components to the total spectral optical depth for a particular scenario, we perform sensitivity studies to analyze the solar flux at the surface in the range 200-1100 nm (which is covered by one of the MetSIS channels) for a wider variety of scenarios. In particular, we define 1000 atmospheric scenarios covering typical and extreme scenarios found at low and mid-latitudes, in which $\tau_{d, 880}$ ranges between 0 and 1.5 , $\tau_{c a, 12.1}$ between 0 and 0.15 , and $N_{\mathrm{O}_{3}}$ between 1 and $3 \mu \mathrm{m}$-atm.

Figure 2 shows the ratio $T / E$ for each scenario when $\mu_{0}=0.85$. A similar behavior has been found for different values of $\mu_{0}$. This ratio is mainly affected by dust optical depth, showing a decrease of about a $20 \%$ between low $(\sim 0.3)$ and high $(\sim 1.5)$ values of $\tau_{d}$. The effect of water ice clouds is very small because both the values and variability of $\tau_{c}$ are much lower than those for $\tau_{d}$, with changes in $T / E$ below $2 \%$ between the extreme scenarios $\left(\tau_{c}=0\right.$ and $\left.\tau_{c}=0.15\right)$. Finally, variations of $\mathrm{O}_{3}$ abundance in the range $1-3 \mu$ m-atm (represented in Fig. 2 by different marker sizes) have a negligible effect on $T / E$, with changes lower than $0.04 \%$.

The described behavior of $T / E$ suggests a method to estimate dust optical depth from MetSIS measurements in a specific band. From a given measurement of $T$, we first calculate the ratio $T / E$, with $E$ given by Eq. (1), and then we use a look-up table generated from model results for different optical depths to find the one that best matches the observations. We complement this method to retrieve dust optical depths with an alternative approach described in Section 4.3.

\subsection{Diurnal evolution of solar fluxes under different scenarios}

Here we characterize the radiative environment at the latitude range of the initially selected MetNet landing sites $\left(30^{\circ} \mathrm{N}-\right.$ $30^{\circ} \mathrm{S}$ ) in the MetSIS spectral range $200-1100 \mathrm{~nm}$. We first analyze the diurnal evolution of the solar flux and the total

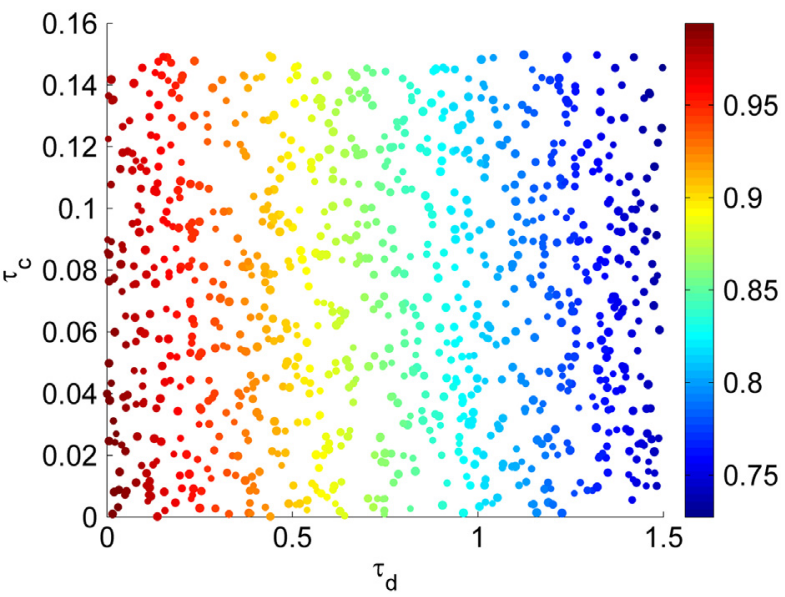

Fig. 2. $T / E$ for 1000 atmospheric scenarios characterized by dust optical depth (horizontal axis), water ice clouds optical depth (vertical axis), and ozone abundance (marker size, which increases with ozone abundance, with values between $1 \mu \mathrm{m}$-atm and $3 \mu \mathrm{m}$ atm).

amount of energy received during one sol (the daily irradiation) as a function of latitude and $\tau$. Then, we compare solar fluxes in the range $200-1100 \mathrm{~nm}$ with the total shortwave radiation. This comparison is important because it allows to estimate the total shortwave flux, which is a key term to calculate the surface energy budget and thus the thermal inertia (Martínez et al. 2014), from in situ measured fluxes between 200 and $1100 \mathrm{~nm}$.

Figure 3 shows the diurnal evolution of the surface solar flux in the 200-1100 $\mathrm{nm}$ band at four different latitudes of $30^{\circ} \mathrm{N}, 10^{\circ} \mathrm{N}, 10^{\circ} \mathrm{S}$, and $30^{\circ} \mathrm{S}$ at the Northern Hemisphere winter solstice $\left(L_{S}=270^{\circ}\right)$. Around this time of the year and at these latitudes, dust optical depths are expected to be high (Lemmon et al. 2015), significantly exceeding the optical depth of water ice clouds (Smith 2004). Thus, we set typical values for these dusty conditions of $\tau_{d, 880}=1, \tau_{c a, 12.1}=0.02$ and surface albedo at $750 \mathrm{~nm} A_{750}=0.25$ (mean value between bright and dark regions of Mars; Mustard \& Bell 1994).

The diffuse radiation $(D)$ is higher than the direct component $(B)$ at the four considered locations, with the ratio direct to diffuse irradiation $(B / D)$ increasing toward the South from 0.23 at $30^{\circ} \mathrm{N}$ to 0.55 at $30^{\circ} \mathrm{S}$. This is expected because the mean photon path between the TOA and the surface increases northward. Further valuable information can be obtained when analyzing the ratio between the diffuse irradiation at the surface and the irradiation at the TOA $(D / E)$. This ratio shows small latitudinal variability, taking values between 0.504 and 0.525 , and thus allowing for a very simple estimation of the diffuse daily irradiation just from the model results at the TOA. We note that for clearer atmospheres, the ratio $D / E$ presents a larger latitudinal variability (between 0.288 and 0.379 for $\tau_{d, 880}=0.3$ ), and thus the diurnal evolution of the diffuse flux at the surface provided by the model is necessary to compute the diffuse daily irradiation at each latitude.

We complement the previous analysis with the study of variations of the surface solar flux between 200 and $1100 \mathrm{~nm}$ at a given latitude due to changes in $\tau$. Figure 4 shows the diurnal evolution of the surface solar flux at an equatorial location $\left(\phi=0^{\circ}\right)$ at $L_{s}=270^{\circ}$ for a range of dust optical depths at $880 \mathrm{~nm}$ between 0 and 4.6, which is the maximum 

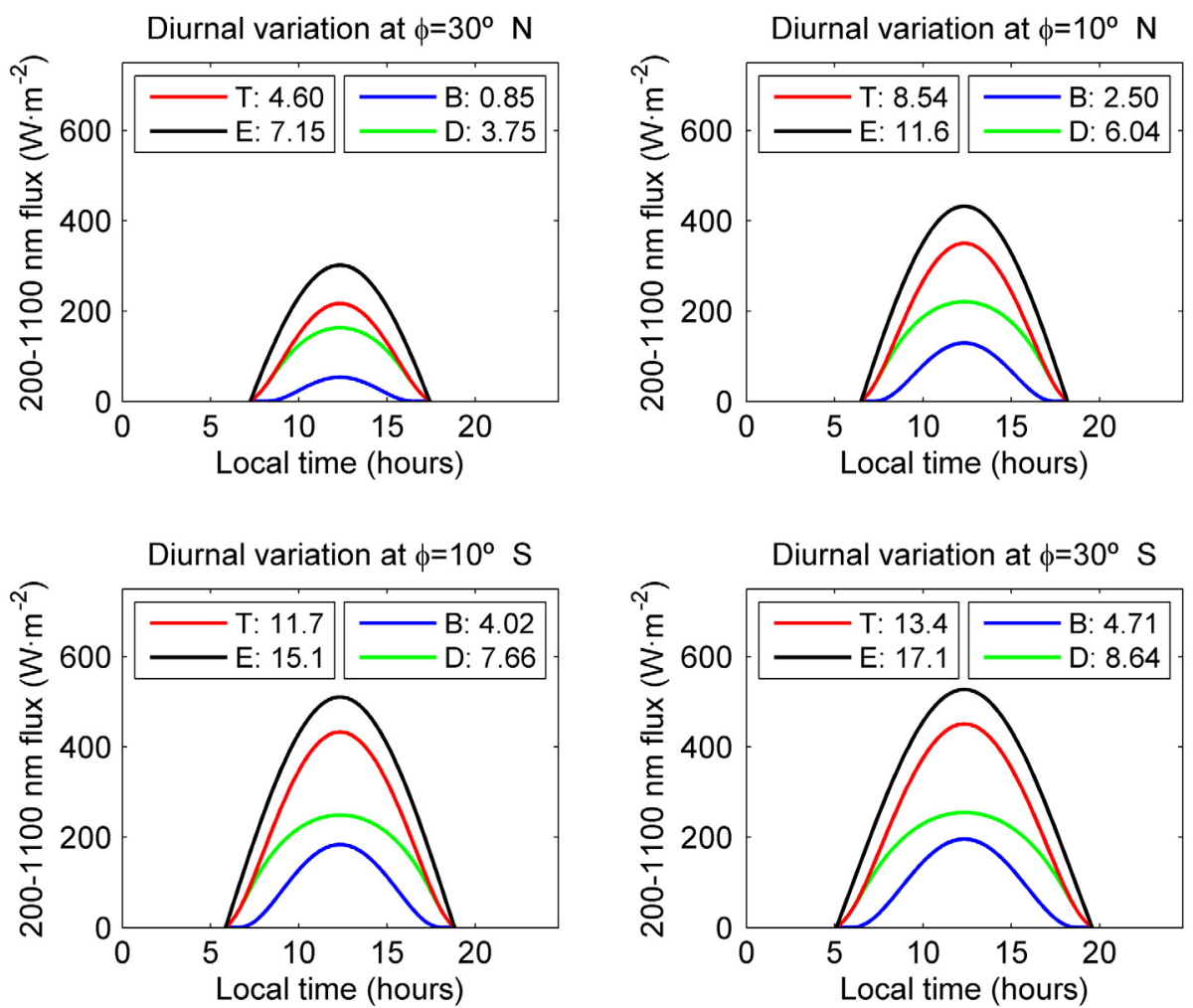

Fig. 3. Diurnal evolution of the direct ( $B$, blue line), diffuse ( $D$, green line), and total ( $T$, red line) surface solar flux between $200 \mathrm{and} 1100 \mathrm{~nm}$ at four different latitudes during the Northern Hemisphere winter solstice, for $\tau_{d, 880}=1$ and $\tau_{c a, 12,1}=0.02$. The flux at the TOA $(E)$ is represented by the black line. The values of the legend represent daily irradiation between 200 and $1100 \mathrm{~nm} \mathrm{in} \mathrm{MJ} \mathrm{m}^{-2}$.
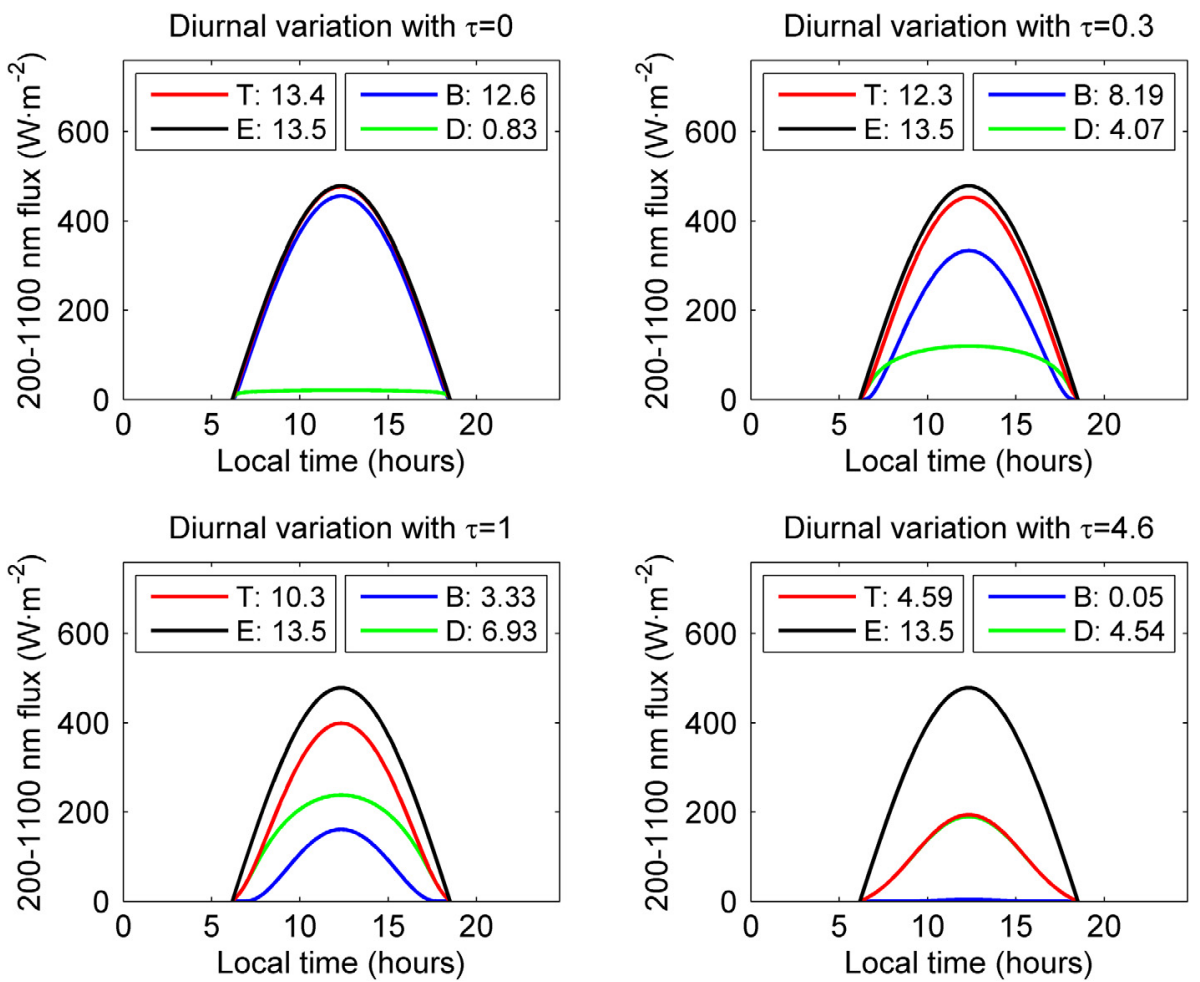

Fig. 4. As in Figure 3, but for different values of $\tau_{d, 880}$ in the range $0-4.6$ at a fixed latitude $\phi=0^{\circ}$. 
Á. Vicente-Retortillo et al.: Modeling solar radiation fluxes on Mars

Diurnal variation at $\phi=30^{\circ} \mathrm{N}$ with $\tau=1$

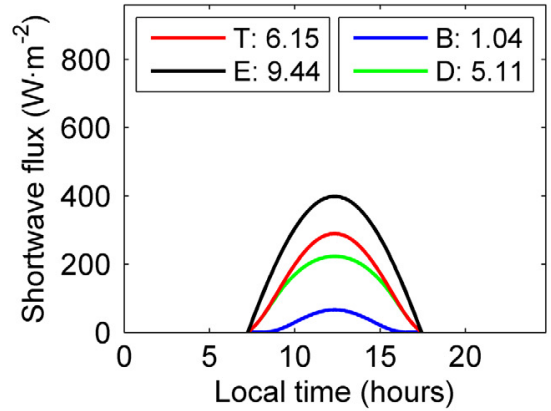

Diurnal variation at $\phi=0^{\circ}$ with $\tau=0.3$

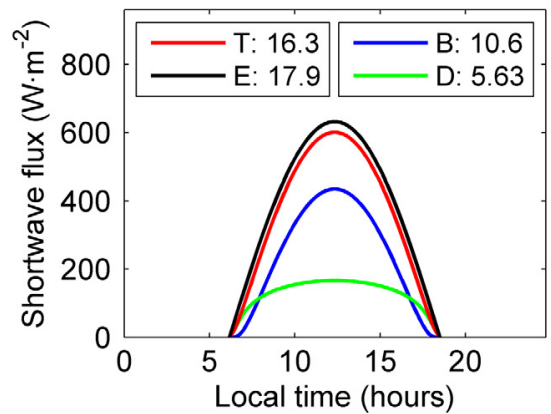

Diurnal variation at $\phi=30^{\circ} \mathrm{S}$ with $\tau=1$

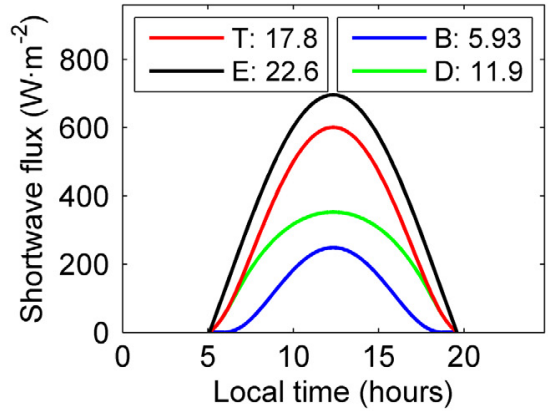

Diurnal variation at $\phi=0^{\circ}$ with $\tau=1$

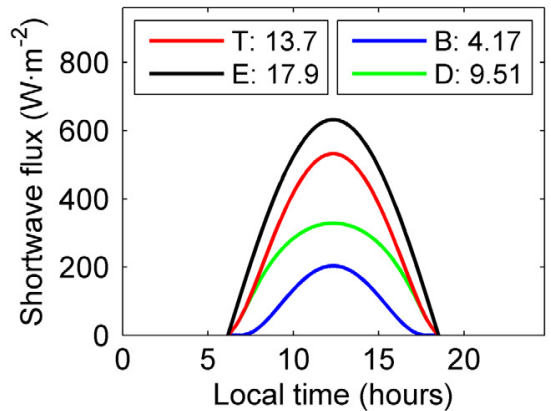

Fig. 5. As in Figure 3, but for the total shortwave flux, and for the combinations of $\phi$ and $\tau_{d, 880}$ specified in the titles.

optical depth observed at the opportunity landing site during the first five Mars years of measurements (Lemmon et al. 2015).

The diurnal evolution of solar radiation at the surface is mainly affected by the amount of dust in the atmosphere, as expected from the sensitivity studies shown in the previous section. In the dust-free scenario, the total irradiation at the surface accounts for about $99 \%$ of its value at the TOA $(T / E=0.99)$, the remaining $1 \%$ being backscattered to space by water ice clouds and molecular gases or being absorbed by gas molecules (the absorption by water ice clouds is negligible in the MetSIS range, as discussed in the previous section). Most of the surface flux is composed by direct radiation $(B / T=0.94)$ due to the small optical depths of water ice clouds and gas molecules, which have a small and a virtually negligible impact between 200 and $1100 \mathrm{~nm}$, respectively, with more than $90 \%$ of the diffuse irradiation caused by the water ice clouds.

When the dust optical depth is 0.3 , which is a typical value under clear conditions at the Spirit landing site (Lemmon et al. 2015), the total irradiation at the surface accounts for about $91 \%$ of its value at the TOA $(T / E=0.91)$. In this case, the ratio between direct and total irradiation $(B / T)$ becomes 0.68 , and the amount of direct radiation is larger than the diffuse one except at and near sunrise and sunset Sun's positions, which is consistent with a longer solar ray path at those times.

Under dusty conditions ( $\tau=1$ ), the ratio between the irradiation at the surface and that at the TOA is $T / E=0.76$. In this case, the diffuse flux is always larger than the direct one, with the $B / T$ ratio showing values of 0.32 , which highlights the important role played by the diffuse radiation in the daily irradiation.
Under very dusty conditions ( $\tau=4.6)$, there is virtually no direct radiation at the surface and $B / T \sim 0.01$. However, since the dust single scattering albedo and the asymmetry factor are relatively high, even under these conditions a significant amount of radiation reaches the surface $(T / E=0.34)$.

We compare now solar fluxes in the range $200-1100 \mathrm{~nm}$ with fluxes in the entire shortwave range. Figure 5 shows the diurnal evolutions of the total shortwave flux and the daily irradiations at $L_{s}=270^{\circ}$ for four of the scenarios shown in Figures 3 and 4: $\phi=30^{\circ} \mathrm{N}$ and $\tau_{d, 880}=1 ; \phi=30^{\circ} \mathrm{S}$ and $\tau_{d, 880}=1$; $\phi=0^{\circ}$ and $\tau_{d, 880}=0.3$; and $\phi=0^{\circ}$ and $\tau_{d, 880}=1$. In each scenario, the ratio of the direct irradiation in the MetSIS range to the total shortwave direct irradiation $\left(B / B_{\mathrm{SW}}\right)$ is higher than $E / E_{\mathrm{SW}}$. This is because the weighted mean extinction efficiency is lower between 200 and $1100 \mathrm{~nm}$ than in the total shortwave range. Therefore, for a given location, $B / B_{\mathrm{SW}}$ increases with optical depth. In particular, $B / B_{\mathrm{SW}}$ increases at the equator from 0.77 when $\tau=0.3$ to 0.89 when $\tau=4$.6. For the same reason, the ratio $B / B_{\mathrm{SW}}$ increases for a given optical depth when the mean solar elevation decreases: For $\tau=1$, its value is 0.818 for $\phi=30^{\circ} \mathrm{N}$ and 0.795 for $\phi=30^{\circ} \mathrm{S}$.

In contrast, $D / D_{\mathrm{SW}}$ shows a weak dependence on the scenario, taking values between 0.724 and 0.734 . The values of $T / T_{\mathrm{SW}}$ at $\phi=0^{\circ}$ decrease as the optical depth increases $\left(T / T_{\mathrm{SW}}=0.755\right.$ when $\tau=0.3$ and $T / T_{\mathrm{SW}}=0.732$ when $\tau=4.6$ ) because the relative contribution of the diffuse component to the total radiation increases with the optical depth. For the three scenarios of Figure 5 with $\tau=1$, the ratio $T / T_{\mathrm{SW}}$ shows a very small variability, taking values between 0.748 at $\phi=30^{\circ} \mathrm{N}$ and 0.750 at $\phi=30^{\circ} \mathrm{S}$ due to changes in the mean solar zenith angle. We have also calculated the ratio $T / T_{\mathrm{SW}}$ for the 1000 scenarios defined in the previous section. As it happened for $T / E$ (Fig. 2), the ratio is controlled 

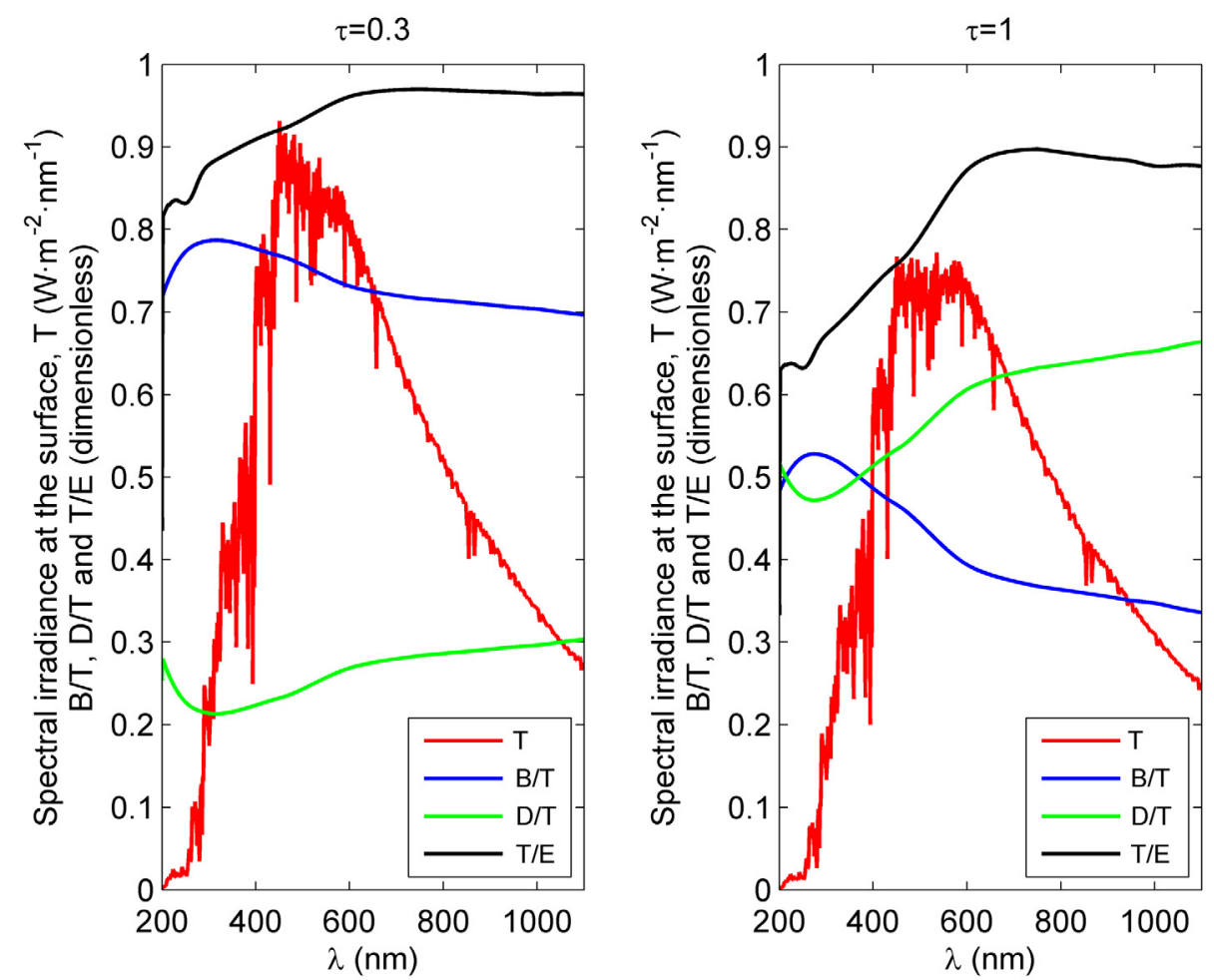

Fig. 6. Total surface spectral irradiance ( $T$, red line), ratios of direct and diffuse irradiances to $T, B / T$ (blue line) and $D / T$ (green line), and ratio of $T$ to the irradiance at the TOA, $T / E$ (black line), between 200 and $1100 \mathrm{~nm}$ for clear (left) and dusty (right) conditions. Simulations run for $\phi=0^{\circ}, L_{s}=270^{\circ}, \tau_{c a, 12.1}=0.02, N_{\mathrm{O}_{3}}=1 \mu \mathrm{m}$-atm, at local noon.

by the dust optical depth. These results show that the shortwave flux can be estimated from the flux between 200 and $1100 \mathrm{~nm}$ and from the model-derived ratios.

\subsection{Spectral irradiance under different scenarios}

In this subsection we complement the study of broadband solar fluxes by analyzing their spectral features between 200 and $1100 \mathrm{~nm}$. Figure 6 shows a comparison between the total spectral irradiance at the surface $(T)$ and the ratios $B / T, D / T$, and $T / E$ for two different values of dust optical depths at $880 \mathrm{~nm}\left(\tau_{d, 880}=0.3\right.$ and $\left.\tau_{d, 880}=1\right)$. In both cases, $\phi=0^{\circ}$, $A_{750}=0.25, L_{s}=270^{\circ}, \tau_{c a, 12.1}=0.02, N_{\mathrm{O}_{3}}=1 \mu \mathrm{m}$-atm, and $t=0$ (which corresponds to noon).

For every wavelength in the range $200-1100 \mathrm{~nm}$, the direct radiation at the surface is larger than the diffuse component when $\tau_{d, 880}=0.3$. However, similar values of diffuse and direct spectral irradiance are found in the UV range when $\tau_{d, 880}=1$, while at longer wavelengths the diffuse component becomes larger than the direct component.

The $D / T(B / T)$ ratio increases (decreases) for every wavelength with dust opacity. However, the qualitative behavior of $D / T$ and $B / T$ is similar for both optical depths. At the shortest wavelengths, $D / T$ decreases toward longer wavelengths mainly due to the decreasing effect of Rayleigh scattering. Then, up to $600 \mathrm{~nm}, D / T$ increases markedly mainly due to the increase in dust single scattering albedo. Between 600 and $1100 \mathrm{~nm}, D / T$ keeps increasing with wavelength due to the increase in the spectral optical depth, but at a smaller rate.

The ratio $T / E$ decreases with increasing values of dust optical depth, and its qualitative behavior is similar for both optical depths. As the dust optical depth increases, the difference between the UV and NIR scattering regimes becomes more apparent, and this causes a more pronounced increase in $T / E$ across visible wavelengths. Therefore, as the dust optical depth increases, the shape of the total spectral irradiance changes, with its peak shifting toward longer wavelengths.

Taking advantage of the spectral behavior of $T / E$ described above, we show an alternative method to retrieve dust optical depth from MetSIS measurements in different spectral bands. While the method shown in Section 4.1 is based on the effect of optical depth on the ratio $T / E$ in a given spectral region, the alternative method described here relies on the effect of optical depth on the differences between the values of $T$ measured in bands with different radiative properties.

Figure 7 shows the ratio between the spectral irradiance at the surface at $440 \mathrm{~nm}$ and at $600 \mathrm{~nm}$, which correspond to the centers of two MetSIS channels, for values of $\tau_{d, 880}$ in the range $0-1.5, \tau_{c a, 12.1}$ in the range $0-0.15$, and $N_{\mathrm{O}_{3}}$ in the range 1-3 $\mu \mathrm{m}$-atm. As in Figure 2, the ratio $T_{440} / T_{600}$ is mainly controlled by dust optical depth because in this spectral range the radiative properties of water ice clouds show a small dependence on wavelength and the variability of $\tau_{c}$ is smaller than that of $\tau_{d}$. The differences in the typical range of optical depths can reach a $20 \%$, with the lowest ratios occurring at high optical depths, being consistent with the behavior of $T / E$ described above. As in the case where only one band is used, a look-up table showing values of the ratio of $T$ at different bands (i.e. 440 and $600 \mathrm{~nm}$ ) is generated from model results for different optical depths to find the one that best matches the observations.

By using both methods to estimate optical depths, we can obtain further information on the particle size distribution because the spectral radiative properties (used to retrieve the 


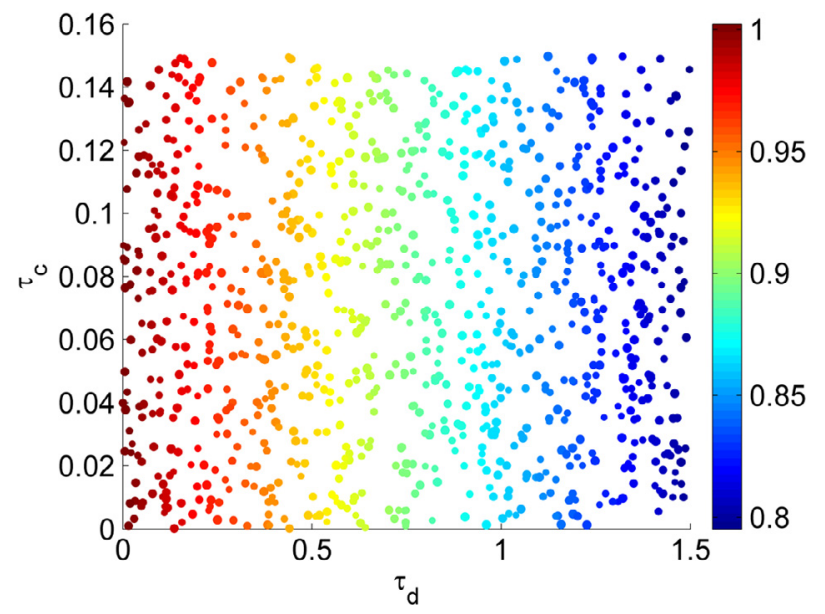

Fig. 7. As in Figure 2, but for the ratio of the spectral irradiance at $440 \mathrm{~nm}$ to that at $600 \mathrm{~nm}, T_{440} / T_{600}$.

optical depth) depend on such distributions. As an example, there is a difference of $4 \%$ between $T_{440} / T_{600}$ using a lognormal dust size distribution with $r_{\text {eff }}=1.5 \mu \mathrm{m}$ and $v_{\text {eff }}=0.3$, and using the same distribution but with $r_{\mathrm{eff}}=1 \mu \mathrm{m}$ and the same $v_{\text {eff }}$ value. Therefore, we can estimate the optical depth by comparing the measurements in different bands with generated look-up tables of $T / E$ for the wider MetSIS band (first method) and $T_{440} / T_{600}$ (second method) for different size distributions, and then identify the size distribution for which the difference between the two optical depths at the reference wavelength is minimum.

Using the model and with some additional information, we can obtain an optical depth from the measurements at other MetSIS channels. As an example, we could use the wider channels in the UVA (315-400 nm) and NIR (700-1100 nm) regions. By knowing the surface pressure, the effect of Rayleigh scattering is considered for the calculation of the UVA flux, and then a value of the optical depth can be obtained from the ratio $T_{\mathrm{UVA}} / T_{\mathrm{NIR}}$. Subsequently, it is possible to compare the results to check the consistency of the retrieved optical depths.

From the results shown in this section we can conclude that useful scientific information, such as temporal variations from diurnal to seasonal time scales of the atmospheric optical depth and particle size distribution, can be obtained from a comparison between measurements and COMIMART results.

\section{Summary and conclusions}

We have developed a comprehensive radiative transfer model to study the solar irradiance that reaches the Martian surface. It includes updated wavelength-dependent radiative parameters for dust, water ice clouds, and gas molecules. The model enables the characterization of the radiative environment under different scenarios in the spectral regions covered by MetSIS and REMS UV sensors.

The model is very versatile as it contains a number of input parameters that can be modified: dust optical depth at $880 \mathrm{~nm}$, water ice clouds opacity at $825 \mathrm{~cm}^{-1}$, abundance of different atmospheric gases, local time, solar longitude, latitude, surface albedo, spectral band and the wavelength-dependent values of extinction efficiency, single scattering albedo, and asymmetry factor. By combining different values of these parameters a wide number of scenarios can be defined, covering the range of conditions that can be found in the Martian atmosphere.

We have validated the model by comparing the calculated surface fluxes to those obtained using the DISORT algorithm, showing an excellent agreement for a wide range of conditions. In addition, we have tested some important assumptions considered in the model, showing their validity in the range between 200 and $1100 \mathrm{~nm}$.

We have shown results of different nature: diurnal evolution of the solar fluxes at the surface (both the direct and diffuse components), spectral irradiance features, and daily irradiation values. We have discussed the effect of the different atmospheric components on the total spectral atmospheric optical depth, and have described two alternative methods to retrieve atmospheric optical depths from in situ measurements. The first considers measured and calculated fluxes in a single band, while the second considers measured and calculated fluxes in different bands with different radiative properties; by combining both methods, additional information about particle size distribution can be obtained. Therefore, the use of the COMIMART model in combination with measurements by the MetSIS sensor aboard the MetNet mission can greatly enhance its scientific return.

Acknowledgements. This work has been partially supported by the research projects AYA2011-29967-C05-02, CGL2011-25327 and EU ERA-NET Plus (PCIN-2014-013-C07-04), and by the Instituto de Matemática Interdisciplinar (IMI) of the Universidad Complutense. The author A. Vicente-Retortillo wishes to acknowledge the Spanish Ministry of Economy and Competitiveness (MINECO) for the granted FPI fellowship (BES-2012-059241). We want to thank M.J. Wolff and an anonymous reviewer for their excellent suggestions to improve the paper. The editor thanks M.J. Wolff and an anonymous referee for their assistance in evaluating this paper.

\section{References}

Berk, A., L.S. Bernstein, G.P. Anderson, P.K. Acharya, D.C. Robertson, J.H. Chetwynd, and S.M. Adler-Golden. MODTRAN cloud and multiple scattering upgrades with application to AVIRIS. Remote Sens. Environ., 65, 367-375, 1998, DOI: $10.1016 /$ S0034-4257(98)00045-5.

Clancy, R.T., M.J. Wolff, and P.R. Christensen. Mars aerosol studies with the MGS TES emission phase function observations: optical depths, particle sizes, and ice cloud types versus latitude and solar longitude. J. Geophys. Res., 108, 5098, 2003, DOI: 10.1029/2003JE002058.

Córdoba-Jabonero, C., L.M. Lara, A.M. Mancho, A. Márquez, and R. Rodrigo. Solar ultraviolet transfer in the Martian atmosphere: biological and geological implications. Planet. Space Sci., 51 (6), 399-410, 2003, DOI: 10.1016/S0032-0633(03)00023-0.

Encrenaz, T., T.K. Greathouse, F. Lefèvre, and S.K. Atreya. Hydrogen peroxide on Mars: observations, interpretation and future plans. Planet. Space Sci., 68 (1), 3-17, 2012, DOI: $10.1016 /$ j.pss.2011.03.019.

Frederick, J.E., and J.E. Mentall. Solar irradiance in the stratosphere: implications for the Herzberg continuum absorption of $\mathrm{O}_{2}$. Geophys. Res. Lett., 9 (4), 461-464, 1982, DOI: $10.1029 /$ GL009i004p00461. 
Gómez-Elvira, J., C. Armiens, L. Castañer, M. Domínguez, M. Genzer, et al. REMS: the environmental sensor suite for the Mars Science Laboratory rover. Space Sci. Rev., 170 (1-4), 583-640, 2012, DOI: 10.1007/s11214-012-9921-1.

Haberle, R.M., C.P. McKay, J.B. Pollack, O.E. Gwynne, D.H. Atkinson, J. Appelbaum, G.A. Landis, R.W. Zurek, and D.J. Flood. Atmospheric effects on the utility of solar power on Mars. In: J.S. Lewis, M.S. Matthews, and M.L. Guerrieri, Editors. Resources of Near-Earth Space, The University of Arizona Press, Tuscon, 845-885, 1993.

Hansen, J.E., and L.D. Travis. Light scattering in planetary atmospheres. Space Sci. Rev., 16, 527-610, 1974.

Henyey, L.G., and J.L. Greenstein. Diffuse radiation in the galaxy. Astrophys. J., 93, 70-83, 1941.

Ityaksov, D., H. Linnartz, and W. Ubachs. Deep-UV absorption and Rayleigh scattering of carbon dioxide. Chem. Phys. Lett., 462 (1), 31-34, 2008, DOI: 10.1016/j.cplett.2008.07.049.

Joseph, J.H., W.J. Wiscombe, and J.A. Weinman. The deltaEddington approximation for radiative flux transfer. J. Atmos. Sci., 33 (12), 2452-2459, 1976, DOI: $10.1175 / 1520-0469(1976) 033<2452$ :TDEAFR $>2.0 . C O ; 2$.

Kahre, M.A., J.R. Murphy, and R.M. Haberle. Modeling the Martian dust cycle and surface dust reservoirs with the NASA Ames general circulation model. J. Geophys. Res., 111, E06008, 2006, DOI: 10.1029/2005JE002588.

Lefèvre, F., F. Montmessin, A. Määttänen, and J.L. Bertaux. The Martian ozone layer as seen by SPICAM: 2004-2011. In: F. Forget, and M. Millour, Editors. The Fifth International Workshop on the Mars Atmosphere: Modelling and Observation, Oxford, UK, 2014, id.3403.

Lemmon, M.T., M.J. Wolff, J.F. Bell III, M.D. Smith, B.A. Cantor, and P.H. Smith. Dust aerosol, clouds, and the atmospheric optical depth record over 5 Mars years of the Mars Exploration Rover mission. Icarus, 251, 96-111, 2015, DOI: $10.1016 /$ j.icarus.2014.03.029.

Lewis, B.R., and J.H. Carver. Temperature dependence of the carbon dioxide photoabsorption cross section between 1200 and 1970 Å. J. Quant. Spectrosc. Radiat. Transfer, 30 (4), 297-309, 1983, DOI: 10.1016/0022-4073(83)90027-4.

Lilensten, J., A.J. Coates, V. Dehant, T.D. De Wit, R.B. Horne, F. Leblanc, J. Luhmann, E. Woodfield, and M. Barthélemy. What characterizes planetary space weather? Astron. Astrophys. Rev., 22 (1), 1-39, 2014, DOI: $10.1007 / \mathrm{s} 00159-014-0079-6$

Lin, C.L., N.K. Rohatgi, and W.B. DeMore. Ultraviolet absorption cross sections of hydrogen peroxide. Geophys. Res. Lett., 5 (2), 113-115, 1978, DOI: 10.1029/GL005i002p00113.

Madeleine, J.-B., F. Forget, E. Millour, L. Montabone, and M.J. Wolff. Revisiting the radiative impact of dust on Mars using the LMD Global Climate Model. J. Geophys. Res., 116, E11010, 2011, DOI: 10.1029/2011JE003855.

Madeleine, J.-B., F. Forget, E. Millour, T. Navarro, and A. Spiga. The influence of radiatively active water ice clouds on the Martian climate. Geophys. Res. Lett., 39, L23202, 2012, DOI: $10.1029 / 2012$ GL053564.

Martínez, G.M., F. Valero, and L. Vázquez. Characterization of the Martian surface layer. J. Atmos. Sci., 66 (1), 187-198, 2009 , DOI: $10.1175 / 2008 J A S 2765.1$.

Martínez, G.M., F. Valero, and L. Vázquez. The TKE budget in the convective Martian planetary boundary layer. $Q$. J. R. Meteorol. Soc., 137 (661), 2194-2208, 2011, DOI: 10.1002/qj.883.

Martínez, G.M., N. Rennó, E. Fischer, C.S. Borlina, B. Hallet, et al. Surface energy budget and thermal inertia at Gale Crater: calculations from ground-based measurements. J. Geophys. Res. [Planets], 119 (8), 1822-1838, 2014, DOI: $10.1002 / 2014 J E 004618$.

Mustard, J.F., and J.F. Bell III. New composite reflectance spectra of Mars from 0.4 to $3.14 \mu \mathrm{m}$. Geophys. Res. Lett., 21 (5), 353-356, 1994, DOI: 10.1029/94GL00198.
Parkinson, W.H., and K. Yoshino. Absorption cross-section measurements of water in the wavelength region 181-199 nm. Chem. Phys., 294 (1), 31-35, 2003, DOI: 10.1016/S0301-0104(03)00361-6.

Patel, M.R., J.C. Zarnecki, and D.C. Catling. Ultraviolet radiation on the surface of Mars and the Beagle 2 UV sensor. Planet. Space Sci., 50 (9), 915-927, 2002,

DOI: 10.1016/S0032-0633(02)00067-3.

Patel, M.R., A. Bérces, T. Kerékgyárto, G. Rontó, H. Lammer, and J.C. Zarnecki. Annual solar UV exposure and biological effective dose rates on the Martian surface. Adv. Space Res., 33 (8), 1247-1252, 2004, DOI: 10.1016/j.asr.2003.08.036.

Perrier, S., J.L. Bertaux, F. Lefèvre, S. Lebonnois, O. Korablev, A. Fedorova, and F. Montmessin. Global distribution of total ozone on Mars from SPICAM/MEX UV measurements. J. Geophys. Res., 111, E09S06, 2006, DOI: $10.1029 / 2006 J E 002681$.

Petrosyan, A., B. Galperin, S.E. Larsen, S.R. Lewis, A. Määttänen, et al. The Martian atmospheric boundary layer. Rev. Geophys., 49 (3), RG3005, 2011, DOI: 10.1029/2010RG000351.

Rannou, P., S. Perrier, J.L. Bertaux, F. Montmessin, O. Korablev, and A. Rébérac. Dust and cloud detection at the Mars limb with UV scattered sunlight with SPICAM. J. Geophys. Res., 111, E09S10, 2006, DOI: 10.1029/2006JE002693.

Read, P.L., and S.R. Lewis. The Martian climate revisited: atmosphere and environment of a desert planet, Springer-Verlag, Berlin, ISBN: 978-3-540-40743-0, 2004.

Rothman, L.S., I.E. Gordon, Y. Babikov, A. Barbe, D.C. Benner, et al. The HITRAN2012 molecular spectroscopic database. J. Quant. Spectrosc. Radiat. Transfer, 130, 4-50, 2013, DOI: 10.1016/j.jqsrt.2013.07.002.

Savijärvi, H., and J. Kauhanen. Surface and boundarylayer modelling for the Mars Exploration Rover sites. O. J. R. Meteorol. Soc., 134 (632), 635-641, 2008, DOI: $10.1002 /$ qj.232.

Savijärvi, H., D. Crisp, and A.-M. Harri. Effects of $\mathrm{CO}_{2}$ and dust on present-day solar radiation and climate on Mars. Q. J. R. Meteorol. Soc., 131, 2907-2922, 2005, DOI: $10.1256 /$ qj.04.09.

Serdyuchenko, A., V. Gorshelev, M. Weber, W. Chehade, and J.P. Burrows. High spectral resolution ozone absorption crosssections - Part 2: temperature dependence. Atmos. Meas. Tech., 7, 625-636, 2014, DOI: 10.5194/amt-7-625-2014.

Smith, M.D. Interannual variability in TES atmospheric observations of Mars during 1999-2003. Icarus, 167 (1), 148-165, 2004, DOI: $10.1016 /$ j.icarus.2003.09.010.

Sneep, M., and W. Ubachs. Direct measurement of the Rayleigh scattering cross section in various gases. J. Quant. Spectrosc. Radiat. Transfer, 92 (3), 293-310, 2005, DOI: $10.1016 /$ j.jqsrt.2004.07.025.

Stamnes, K., S.C. Tsay, W. Wiscombe, and K. Jayaweera. Numerically stable algorithm for discrete-ordinate-method radiative transfer in multiple scattering and emitting layered media. Appl. Opt., 27, 2502-2509, 1988, DOI: $10.1364 / A O .27 .002502$.

Stamnes, K., S.C. Tsay, W. Wiscombe, and I. Laszlo. DISORT, a general-purpose FORTRAN program for Discrete-OrdinateMethod radiative transfer in scattering and emitting layered media: documentation of methodology, version 1.1. ftp:// climate1.gsfc.nasa.gov/wiscombe/Multiple_Scatt/DISORTReport1. 1.pdf, 2000

Vázquez, L., M.P. Zorzano, and S. Jiménez. Spectral information retrieval from integrated broadband photodiode Martian ultraviolet measurements. Opt. Lett., 32 (17), 2596-2598, 2007, DOI: $10.1364 /$ OL.32.002596.

Warren, S.G. Optical constants of ice from the ultraviolet to the microwave. Appl. Opt., 23 (8), 1206-1225, 1984, DOI: 10.1364/AO.23.001206.

Weber, M.J. Handbook of optical materials, CRC Press, Boca Raton, Florida, ISBN: 0-8493-3512-4, 2003. 
Á. Vicente-Retortillo et al.: Modeling solar radiation fluxes on Mars

Wolff, M.J., and R.T. Clancy. Constraints on the size of Martian aerosols from Thermal Emission Spectrometer observations. J. Geophys. Res., 108, 5097, 2003, DOI: 10.1029/2003JE002057. Wolff, M.J., M.D. Smith, R.T. Clancy, R. Arvidson, M. Kahre, F. Seelos IV, S. Murchie, and H. Savijärvi. Wavelength dependence of dust aerosol single scattering albedo as observed by the Compact Reconnaissance Imaging Spectrometer. J. Geophys. Res., 114, E00D04, 2009, DOI: 10.1029/2009JE003350.
Wolff, M.J., R.T. Clancy, J.D. Goguen, M.C. Malin, and B.A. Cantor. Ultraviolet dust aerosol properties as observed by MARCI. Icarus, 208 (1), 143-155, 2010,

DOI: $10.1016 /$ j.icarus.2010.01.010.

Zorzano, M.P., L. Vázquez, and S. Jiménez. Retrieval of ultraviolet spectral irradiance from filtered photodiode measurements. Inverse Prob., 25 (11), 115023, 2009, DOI: $10.1088 / 0266-5611 / 25 / 11 / 115023$

Cite this article as: Vicente-Retortillo Á, Valero F, Vázquez L \& Martínez GM. A model to calculate solar radiation fluxes on the Martian surface. J. Space Weather Space Clim., 5, A33, 2015, DOI: 10.1051/swsc/2015035. 